\title{
High resolution fabrication in chalcogenide glasses
}

\author{
Elisa Nicoletti ${ }^{1}$, Douglas Bulla ${ }^{2}$, Barry Luther-Davies ${ }^{2}$, and Min $\mathbf{G u}^{1}$ \\ ${ }^{I}$ Centre for Micro-Photonics \& CUDOS, Faculty of Engineering of Industrial Sciences, Swinburne University of Technology \\ ${ }^{2}$ Laser Physics Centre \& CUDOS, Research School of Physical Sciences and Engineering, Australian National University. \\ Email:ENicoletti@swin.edu.au
}

\begin{abstract}
Here we report on the theoretical and experimental study of the influence of the laser repetition rate on the direct laser fabrication in chalcogenide glasses (ChGs). Through a numerical model of cumulative heating the optimum conditions for high resolution fabrication in $\mathrm{As}_{2} \mathrm{~S}_{3}$ were found. Nanowires with dimensions down to $\approx \lambda / 12$ have been for the first time successfully fabricated in ChGs.
\end{abstract}

\section{Introduction}

In the past few years a new technology based on direct laser writing (DLW) of photonic structures with ultrashort laser pulses has been demonstrated and has attracted a lot of interest [1-6]. Besides the traditional polymeric photoresist, DLW has been successfully applied to high refractive index and highly nonlinear chalcogenide glasses (ChGs), such as arsenic trisulphide $\left(\mathrm{As}_{2} \mathrm{~S}_{3}\right)$. Most experiments have been carried out using low repetition rate (RR) $(1 \mathrm{kHz})$ Ti:sapphire laser $[2,3]$. Using a femtosecond laser beam it is possible to induce structural modification in the glass through a two-photon induced nonlinear process (my paper). However similar phenomena can also be induced by thermal effects.

Here we report on the direct laser writing fabrication of microstructures in $\mathrm{As}_{2} \mathrm{~S}_{3}$ films using a high repetition rate (RR) ultrafast pulsed laser. The laser RR was found to have significant impact on the structural modifications of ChGs that in turn affects the nanofabrication inside such glasses.

Using a thermal diffusion model we compared the heating accumulation in the material induced by a laser beam at three different repetition rates $(1 \mathrm{KHz}, 82 \mathrm{MHz}$ and $1.6 \mathrm{MHz})$. The theoretical calculations show that $1.6 \mathrm{MHz}$ gives the optimum condition for high resolution fabrication, combining the advantage of the thermal effect while avoiding an excess accumulation of heat close to the focal spot. Experimental results confirmed our prediction and for the first time lines with resolution of $\approx \lambda / 12(68 \pm 14 \mathrm{~nm})$ were fabricated in $\mathrm{As}_{2} \mathrm{~S}_{3}$ glass.

\section{Numerical model of cumulative heating}

The mechanism by which femtosecond laser radiation induces structural changes in a photosensitive material, as well as the extent to which the optical characteristics change, are largely determined by the fabrication parameters and the properties of the material. It has been demonstrated how, using a low RR laser beam, a multi-photon absorption process results in local ionisation, and thus structural changes of the material in the focal region [2].

In this work, a variable RR femtosecond laser was applied for the first time to uncover the relative contributions of thermal diffusion and heat accumulation in microstructures fabrication in $\mathrm{As}_{2} \mathrm{~S}_{3}$ films. To verify and study the dynamics of the cumulative heating, a finite-difference thermal diffusion model was applied to the different fabrication conditions $[7,8]$.

To investigate if an accumulation of heat occurs during the fabrication, we studied the two extreme condition of $1 \mathrm{KHz}$ and $82 \mathrm{MHz}$, as well as an intermediate value of $1.6 \mathrm{MHz}$. From the comparison of the thermal parameters at different RRs we can predict that a high RR will allow for the fabrication of smooth and homogeneous rods at higher scanning speed. In particular DLW at $1.6 \mathrm{MHz}$ is expected to offer the optimum fabrication conditions in $\mathrm{As}_{2} \mathrm{~S}_{3}$ films.

\section{Experimental results}

The investigation on the influence of the laser RR on the fabrication conditions was conducted by fabricating twodimensional (2D) lines at different laser powers and different scanning speeds.

Femtosecond laser pulses operating at $800 \mathrm{~nm}$ were focused into an $\mathrm{As}_{2} \mathrm{~S}_{3}$ film of $\sim 16-20 \mu \mathrm{m}$ in thickness with an oil immersion objective (Olympus, numerical aperture 1.4, $100 \times$ ). An ultrashort pulsed laser (Spectra Physics, Tsunami) with a $10 \mathrm{~W}$ pump laser was used. The pulse width and the RR of the laser were $80 \mathrm{fs}$ and $82 \mathrm{MHz}$ respectively. A Conoptics pulse picker was used to tuned the RR of the laser beam as desired. Suspended lines were fabricated with powers ranging between 300 and $900 \mu \mathrm{W}$ and at 2 different scanning speed $(100 \mu \mathrm{m} / \mathrm{sec}$ and 200 $\mu \mathrm{m} / \mathrm{sec}$ ) (Fig 1(a) inset). The unexposed glass was removed using a solution of diisopentylamine (Alfa Aesar) and DMSO (Sigma-Aldrich) (2-3 mol \%) for about 5-10 minutes. 


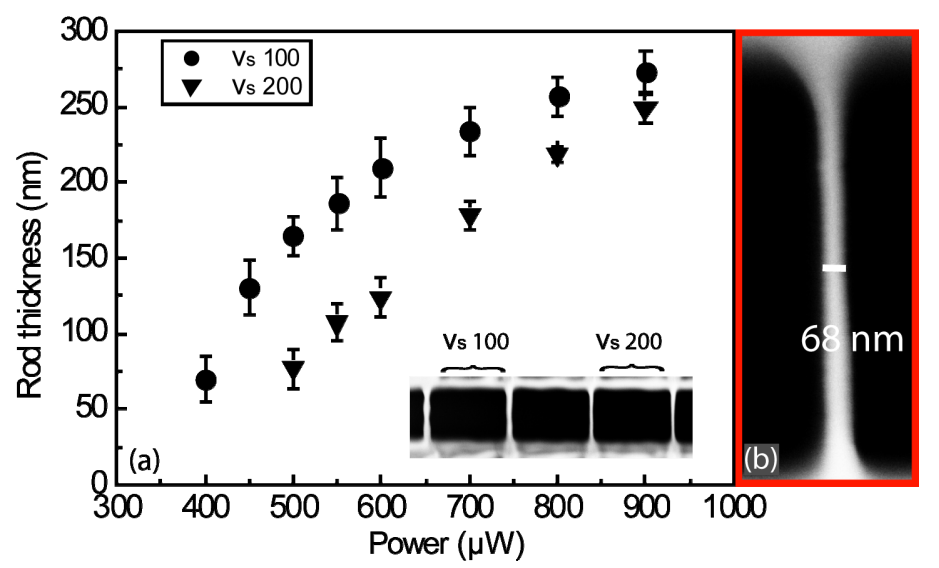

Fig. 1 (a) Experimental plot of the lateral rod dimension versus the laser power for 2different scanning speeds $\left(\mathrm{v}_{\mathrm{s}}\right)(100 \mu \mathrm{m} / \mathrm{sec}$ and $200 \mu \mathrm{m} / \mathrm{sec}$. In the inset a SEM image of the $2 \mathrm{D}$ lines fabricated at constant power $(50 \mu \mathrm{W})$ but at different scanning speeds. Two lines for each scanning speed were fabricated to assure reproducibility In the inset a SEM image of a line fabricated at the threshold power. (b) SEM image of a line fabricated using a laser power of $450 \mu \mathrm{W}$ and a scanning speeds of $200 \mu \mathrm{m} / \mathrm{sec}$.

Fig. 1(a) shows a plot of the lateral the lateral line width as a function of the laser power. The experimental threshold thickness limit shows the possibility to break the current fabrication resolution limit in ChGs. For the first time lines with a resolution of $\approx \lambda / 12$ were fabricated in $\mathrm{As}_{2} \mathrm{~S}_{3}$ glass. Fig. 1(b) shows a SEM image of a line fabricated at a power of $450 \mu \mathrm{W}$ and at a scanning speed of $150 \mu \mathrm{m} / \mathrm{sec}$. The later rod dimension is only $68 \pm 14 \mathrm{~nm}$. All the fabricated rods are smooth and homogeneous and with strong mechanical properties.

This achievement is an important step towards the realisation of all-optical devices. Such nanowires, provide the maximal confinement of light for index guiding structures enabling large enhancements in nonlinear interactions as well as group-velocity dispersion engineering [9]. The effective nonlinearity of ChG photonic nanowires is more than 3 orders of magnitude larger than silica glass nanowires, which promises the possibility to use more readily achievable power levels for nonlinear optical applications and photonics integration devices [9].

Under the 1.6 MHz RR fabrication conditions, 3D PhCs were successfully fabricated and a stop gap observed. For a woodpile structure with lattice parameters $\mathrm{dx}=1 \mu \mathrm{m}$ and $\mathrm{dz}=0.6 \mu \mathrm{m}$ fabricated at a laser power of $550 \mu \mathrm{W}$ and a scanning speed of $100 \mu \mathrm{m} / \mathrm{sec}$, the FTIR spectrum shows a $42 \%$ suppression of the transmission at a wavelength of $2.9 \mu \mathrm{m}$. The mechanical properties of the written structures are preserved proving the possible extension of the DLW fabrication at $1.6 \mathrm{MHz}$ to $3 \mathrm{D}$ microstructures.

\section{Conclusions}

The fabrication conditions in chalcogenide glass were found to be sensitive to the RR of the laser beam. Exploiting the accumulation of heat at the focal point produced by a higher RR was found to be beneficial in terms of features dimensions and surface quality. To better understand the processes that lead to the structural changes in the material, a numerical model of cumulative heat was applied to the fabrication conditions. The calculations indicated $1.6 \mathrm{Mhz}$ as the ideal RR to use for high resolution fabrication. In fact, it facilitates the benefits of the heating effect while avoiding overheating of the glass around the focal spot. With the implementation of a pulse picker in the fabrication system it was possible to obtain rods with lateral dimension of $68 \pm 14 \mathrm{~nm}(\approx \lambda / 12)$ with strong mechanical properties. This is a great achievement for the advancement of optical technologies as the confinement of the light in a small volume contributes to the enhancement of the nonlinear properties of the material. Nonlinear nanowires have recently attracted a lot of interest due to their unique properties, and are intriguing for the fabrication of nanophotonic components and circuits.

\section{References}

1. J. Serbin and M. Gu, "Experimental evidence for superprism effects in three-dimensional polymer photonic crystals" Advanced materials 18, 221-224 (2006).

2. E. Nicoletti, G. Zhou, B. Jia, M. J. Ventura, D. Bulla, B. Luther-Davies, and M. Gu, "Observation of multiple higher-order stopgaps from three-dimensional chalcogenide glass photonic crystals," Optics Letters 33, 2311-2313 (2008).

3. S. Wong, M. Deubel, F. Pérez-Willard, S. John, G. A. Ozin, M. Wegener, and G. Von Freymann, "Direct laser writing of threedimensional photonic crystals with a complete photonic bandgap in chalcogenide glasses" Advanced Materials 18, 265-269 (2006). 
4. M. Straub and M. Gu, "Near-infrared photonic crystals with higher-order bandgaps generated by two-photon photopolymerizatioN" Optics Letters 27, 1824-1826 (2002).

5. M. Straub, M. Ventura, and M. Gu, "Multiple higher-order stop gaps in infrared polymer photonic crystals" Physical Review Letters 91(2003).

6. H. B. Sun, T. Kawakami, Y. Xu, J. Y. Ye, S. Matuso, H. Misawa, M. Miwa, and R. Kaneko, "Real three-dimensional microstructures fabricated by photopolymerization of resins through two-photon absorption" Optics Letters 25, 1110-1112 (2000).

7. Y. Jaluria and S. N. Atluri, "Computational heat transfer" Computational Mechanics 14, 385-386 (1994).

8. A. H. Nejadmalayeri and P. R. Herman, "Rapid thermal annealing in high repetition rate ultrafast laser waveguide writing in lithium niobate" Optics Express 15, 10842-10854 (2007).

9. M. A. Foster, A. C. Turner, M. Lipson, and A. L. Gaeta, "Nonlinear optics in photonic nanowires" Opt. Express 16, 1300-1320 (2008). 